\title{
Use of Exponential Functions in the Evaluations of Stochastic Variables in the Ionizing Radiation Field
}

\author{
Terman Frometa-Castillo ${ }^{1}$ \\ ${ }^{1}$ Oncology Hospital of Santiago of Cuba \\ Correspondence: Terman Frometa-Castillo, 6134 N Oakley Ave Unit 2, Chicago, IL, 60659, USA. Tel: $312-687-$ \\ 6422. E-mail:terman.frometa@gmail.com
}

Received: January 15, 2018

Accepted: March 14, 2018

Online Published: May 31, 2018

doi:10.5539/apr.v10n3p32

URL: https://doi.org/10.5539/apr.v10n3p32

\begin{abstract}
To show that some deficiencies arisen can occur as result of owing to the stochastic processes/effects (SP/Es) in areas of ionizing radiations have not been probabilistically treated nor modeled, because they use exponential functions (EFs) derived from unnecessary differential equations (DEs) or unnecessary definition; and 2) to discuss some statistical models project (SMp) proposals of new probabilistic functions (PFs) that have probabilistic foundations, and will overcome the quoted problems.

The following results were obtained: 1) Determination of deficiencies due to use of EFs in evaluations of the following SP/Es: cell survival attenuation of radiation, radioactive decay and radioactivity; and 2) The SMp formulations for these SP/Es.

The previous SP/Es have not been probabilistically treated nor modelled, since they use EFs that are non-PFs, and some of them are derived from unnecessary solutions of DEs or unnecessary definition. These differential equations used in the derivations do not represent physic properties of the SP/Es, but are simply a mathematic property of the EFs modelling their respective SP/Es. The SMp proposes PFs that will be able to model SP/Es with simple and homogeneous functions using the three types of SP/Es. The SMp models will represent new PFs, where the probability of a random variable $X$ is expressed as $P_{X}=p(y)$, instead of $x$; and the stochastic region is limited by two $0 \%$ and/or $100 \%$-deterministic regions. The SMp treats the radioactivity as a SP/E SMp type P2, and considers there is no need of its current definition.
\end{abstract}

Keywords: LQ S(d) model; attenuation of radiation; radioactive decay; radioactivity; stochastic process

\section{Introduction}

It is very important to understand that, thus far, except the probabilistic treatments of the tumor control probability and normal tissue complication probability (respectively TCP and NTCP), many stochastic processes/effects (SP/Es) in the areas of ionizing radiations have neither been probabilistically treated nor modeled by other researchers, which has led to the following deficiencies: a) Use of a heterogeneous group of complex-deficient or improper models and complex-mathematic parameters; and b) some SP/Es have not been called with probabilistic names.

Despite cell survival (S), radioactive decay, attenuation of radiation, and radioactivity being recognized as SP/Es, these have not been appropriately modeled with probabilistic functions (PFs). Nowadays, these SP/Es have been modeled with non-PFs that have some similarities with PFs. Exponential functions are used for modeling these $\mathrm{SP} /$ Es. Except the S, the others were derived from unnecessary-mathematic procedures; while the derivation of the radioactivity required an unnecessary definition.

The statistical models project (SMp) has classified the behaviors of the mean values of the following SP/Es into three types (P1, P2 and P3). P1: For values of the independent variable $<$ a threshold value (TV) the process is $0 \%$-deterministic, i.e. it will never occur. For values $\geq$ this threshold, the process is increased when the independent variable (IV) increases, from $0 \%$ to $100 \%$, and is stochastic until a determined value of IV where the process becomes $100 \%$-deterministic, i.e. it certainly occurs; P2: For values of IV < a TV, the process is $0 \%$-deterministic; for values $\geq$ this threshold, the process is increased when IV increases, from $0 \%$ to a maximum value, and later it begins to decrease until $0 \%$, at this point the process is $0 \%$-deterministic when IV increases; and P3: For values of IV $<$ a TV, the process is $100 \%$-deterministic; with an increase of IV, it is stochastic and decreases from $100 \%$ to $0 \%$, at this point the process is $0 \%$-deterministic when IV increases. In fact, P3 is a complement of P1. 
The SMp models are PFs capable of describing many SP/Es, which allows simplifying, being more accurate, unifying the treatments and models, and avoiding the current heterogeneous groups of metrics and models based on non-PFs.

\section{Material and Methods}

Radiobiological-probabilistic studies on some current radiobiological concepts and models using exponential functions (EFs), which allowed determining that some SP/Es in the areas of the ionizing radiations have not been probabilistically treated nor modeled by other researchers, because deficient and non-PFs are used. On the hand, based on the three types of SP/Es, the SMp has formulated simple PFs with advantageous parameters that will overcome the problems generated by inappropriate treatments to these SP/Es.

\subsection{The LQ S(d) Model}

The radiosensitivity studies have provided information about cell survival (S), a complement of the cell kill (K), i.e., probabilistically $S=1-K$. The $S$ has been modeled by the linear-quadratic (LQ) model as

$$
L Q S(d)=\exp \left(-\alpha d-\beta d^{2}\right)
$$

Where $\quad \alpha$ and $\beta$ : Mathematic parameters, $d$ : Absorbed dose in one fraction

The LQ S(d) model and LQ S(n,D) formalism ( $D$ is absorbed dose in $n$ fractions, $D=n d$ ) are related as LQ S(n,D) $=(\mathrm{LQ} S(\mathrm{~d}))^{\wedge} \mathrm{n}$. The LQ model and LQ formalism are widely used in the radiobiological field, as is shown in (American Association of Physicists in Medicine [AAPM], 2012) and (Jones \& Al Morgan, 2007)

The LQ model formulation did not use mathematic procedure. This is not entirely appropriate for describing $\mathrm{S}$ because its values are in the semi-open interval $[0 ; 1), S=1$ in $d=0$, and $S=0$ in $d=+\infty$. These results do not reflex the reality, and are the same for any tissue $\mathrm{T}$ and radiation R. Nonetheless, the usefulness of this model is conditioned by its similarities with the PFs, such as, $\mathrm{S}$ continuously decreases when $d$ increases.

The radiation interactions with living tissues can produce cell kill (K), cell sub-lethal damage (SL), and undamaged cells (U). These are stochastic effects; and there will be a better understanding, if these are renamed with probabilistic concepts, such as $\mathrm{K}$ with cell kill probability (CKP), SL with cell sub-lethal damage probability (CSLDP), and U with undamaged cells probability (UCP). The CKP will represent the mean quantity of cells that will be killed divided by total number of cells when same volumes of tissues are independently irradiated with a dose $d$ in various experiments, while CSLDP and UCP will be associated respectively to sub-lethal damaged and undamaged cells.

\subsection{The Current Radioactive Decay Expression}

Although currently radioactive decay has been recognized as a stochastic process, this has not been probabilistically treated. Equation (3) is currently used for determining the number of radioactive nucleus $\mathrm{N}(\mathrm{t})$ in $t$, for which the following differential equation has been employed, where $\lambda$ is the decay constant (International Atomic Energy Agency [IAEA], 2003).

$$
\frac{d N}{d t}=-\lambda N
$$

The radioactive decay is modeled as

$$
\frac{N(t)}{N 0}=e^{-\frac{\ln (2) t}{\frac{T 1}{2}}}
$$

Where $\mathrm{N} 0: \mathrm{N}(\mathrm{t})$ in $\mathrm{t}=0, \mathrm{~T}_{1 / 2}$ : Half-life $\left(\lambda=\ln (2) / T_{1 / 2}\right), t$ : Time

This model is not completely appropriate for describing the $\mathrm{N}(\mathrm{t}) / \mathrm{N} 0$, because its values are in the semi-open interval $[1 ; 0), \mathrm{N}(\mathrm{t}) / \mathrm{N} 0=1$ in $\mathrm{t}=0$, and $\mathrm{N}(\mathrm{t}) / \mathrm{N} 0=0$ in $\mathrm{t}=+\infty$. These results do not reflex the reality, and are the same for any radioactive source. Nonetheless, the usefulness of this model is conditioned by its similarities with the PFs, where $\mathrm{N}(\mathrm{t}) / \mathrm{N} 0$ continuously decreases when $t$ increases.

\subsection{The Current Attenuation of Radiation Expression}

Although the attenuation of radiation (AR) has been currently recognized as a stochastic process, this has not been treated probabilistically. Equation (5) is currently used for determining the intensity of radiation $I(x)$ absorbing a 
material of thickness $x$ at an initial intensity I0. For obtaining the AR expression, the following differential equation was employed, where $\mu$ is the linear attenuation coefficient (Parks, 2001).

$$
\frac{d I}{d x}=-\mu I
$$

Nowadays, the AR is modeled with the solution of this equation as

$$
\frac{I(x)}{I 0}=e^{-\frac{\ln (2) x}{X_{1} / 2}}
$$

Where $I 0: I(x)$ for $x=0, \mathrm{X}_{1 / 2}$ : Half-value thickness $\left(\mu=\ln (2) / X_{1 / 2}\right), x$ : Thickness

This model is not completely appropriate for describing $\mathrm{I}(\mathrm{x}) / \mathrm{I} 0$, because its values are in the semi-open interval [1; $0), \mathrm{I}(\mathrm{x}) / \mathrm{I} 0=1$ in $\mathrm{x}=0$, and $\mathrm{I}(\mathrm{x}) / \mathrm{I} 0=0$ in $\mathrm{x}=+\infty$. These results do not reflex the reality, and are the same for any source of radiation and material. Nonetheless, the usefulness of this model is conditioned by its similarities with the PFs, such as I(x)/I0 continuously decreases when $x$ increases.

\subsection{The Current Radioactivity Expression}

The current expression for the radioactivity is derived from :"The activity $A(t)$ of a radioactive substance at time $t$ is defined as the product of $\lambda$ and the number of radioactive nuclei $N(t)$ " (IAEA, 2003) i.e.,

$$
A(t)=\lambda N(t)
$$

Using Eq. (3) and Eq. (6), the $\mathrm{A}(\mathrm{t})$ expression is written as

$$
A(t)=A 0 e^{-\frac{\ln (2) t}{T 1 / 2}}
$$

Where $A 0: A(t)$ in $t=0, \mathrm{~T}_{1 / 2}$ : Half-life, t: Time

Equations (1), (3), (5), and (7) are non-PFs, and have similarities with the PFs. Except Eq. (1) with its direct formulation, the others were determined from unnecessary differential equations, or unnecessary definition. Equation (2) has been considered as a reference for the mathematical approach adopted and the result obtained from Eq. (4) (Basic Physics of Nuclear Medicine/Attenuation of Gamma-Rays, 2018).

\section{Results and Discussion}

The probability is the extent to which an event is likely to occur, measured by the ratio of the favorable cases to the whole number of cases possible, or ratio of the number of something resulting of an effect or process and its initial number. The probability of random variables $\mathrm{X}\left(\mathrm{P}_{\mathrm{X}}\right)$ for many processes/effects $(\mathrm{P} / \mathrm{Es})$ in areas of the ionizing radiations are stochastic, because the same value of its independent variable $y$ belonging to stochastic region $\mathrm{P}_{\mathrm{X}}$ can take values within a determined range as result of several occurrence of the $\mathrm{P} / \mathrm{E}$. For a value $y$ in the deterministic regions, $\mathrm{P}_{\mathrm{X}}$ will be $0 \%$ or $100 \%$. These $\mathrm{P} / \mathrm{Es}$ should be modeled with PFs. There is a representation and description of uncertainties of the SP/Es SMp type P1 in (Frometa-Castillo \& Frometa-Leon, 2017) and they are used in the SMp NTCP models of (Frometa-Castillo, 2017a)

\subsection{Applications of the SMp type P1}

Based on the SP/Es SMp type P1, the SMp has formulated the model for the cell kill (K) as (Frometa-Castillo, 2017b).

$$
\operatorname{SMp} K(d)=\left(\frac{d-d \min K}{d \max K-d \min K}\right)^{p K} \quad \operatorname{dmin} K \leq d \leq d \max K
$$

Where $d \min K$ : Threshold for $\mathrm{K}, d \max K$ : Minimum dose for $\mathrm{K}=100 \%, p K$ : Power of this model $(p K>0)$

$d$ : Dose in a fraction

In $d<\operatorname{dmin} K$ and $d>d \max K, \mathrm{~K}(\mathrm{~d})$ is respectively the $0 \%$ - and $100 \%$-deterministic effect.

$K$ continuously increases when $d$ increases in this model, takes values in the closed interval [0;1], and defines the deterministic regions for each determined tissue $\mathrm{T}$ and radiation $\mathrm{R}$. Its parameters can be obtained from the cell survival information; however, when data are not available, these can be derived from the similarities between SMp S(d) and LQ S(d) models. The $L Q S\left(d ; \alpha=0.307 G y^{-1}, \alpha / \beta=10 G y\right)$ has similarity with the model $1-S M p K(d$; $d \min K=0.2 G y, \operatorname{dmax} K=8 G y, p K=0.4)$. 
Given the LQ S(d) used nowadays is an EF, which has similarities with the PFs, in particular the SMp S(d) = 1SMp K(d), the SMp model can be used in whatever situations employing LQ S(d), and even replace this model. The use of the SMp model will improve, as is shown in the comparison between the LQ S(d) and the SMp K(d) in Table 1, where the differences represent the deficiencies of the LQ S(d) model.

Table 1. Similarities and differences between the LQ S(d) and the SMp K(d)

\begin{tabular}{|c|c|c|c|}
\hline Aspect & $L Q S(d)$ & $\operatorname{SMp~} K(d)$ & Remarks \\
\hline $\begin{array}{l}\text { Behavior in function of the } \\
\text { independent variable in the } \\
\text { stochastic region }\end{array}$ & $\begin{array}{l}\text { Continuously } \\
\text { decreases }\end{array}$ & Continuously increases & $\begin{array}{l}\text { The SMp K(d) has a behavior typical of a SMp } \\
\text { type P1, and the LQ S(d) own of a complement } \\
\text { of the former. }\end{array}$ \\
\hline $\begin{array}{l}\text { Definition of the stochastic } \\
\text { region; i.e. the interval }\end{array}$ & Not clearly defined & Well defined & $\begin{array}{l}\text { In the LQ } \mathrm{S}(\mathrm{d}) \text { always } \mathrm{dminK}=0 \text {, while } \mathrm{dmaxK} \\
\text { can be obtained by estimation. The SMp }\end{array}$ \\
\hline$[d \min K ; \operatorname{dmax} K]$ & & & $\begin{array}{l}\text { defines an interval for each tissue } \mathrm{T} \text { and } \\
\text { radiation } \mathrm{R}\end{array}$ \\
\hline Parameters & $\begin{array}{l}\alpha \text { and } \beta \text { are } \\
\text { mathematical }\end{array}$ & $\begin{array}{l}d \min K \text { and } d \max K \text {, two } \\
\text { radiobiological, and } p K \\
\text { mathematical }\end{array}$ & $\begin{array}{l}\text { The SMp parameters have a major } \\
\text { familiarization }\end{array}$ \\
\hline Probabilistic foundation & None & Strong & The LQ S(d) is based on math foundation \\
\hline Radiobiological foundation & It is not good & Very acceptable & $\begin{array}{l}\text { In the LQ } \mathrm{S}(\mathrm{d}) \text {, the } S=1 \text { in } \mathrm{d}=0 \text { and } \mathrm{S}=0 \text { in } \\
\mathrm{d}=+\infty \text {, and these values are the same for } \\
\text { whatever tissue } \mathrm{T} \text { and radiation } \mathrm{R}\end{array}$ \\
\hline Math complexity & Middle & Low & \\
\hline
\end{tabular}

\subsection{Application of the SMp type P3}

The radioactive decay can be treated as a stochastic process SMp type P3 function of the time $t$, so its SMp model is written as

$$
\operatorname{SMp} N(t) / N 0=\left(\frac{t \max -t}{\operatorname{tmax}-t \min }\right)^{p N t} \quad t \min \leq t \leq t \max
$$

Where $N 0: N(t)$ in $t=0$, tmin: Maximum value of $t$ for $\mathrm{N}(\mathrm{t}) / \mathrm{N} 0=100 \%$, tmax or $T f$ : The life time or minimum value of $t$ for $\mathrm{N}(\mathrm{t}) / \mathrm{N} 0=0 \%, p N t$ : Power of this model $(p N t>0), t$ : Time

In $t<$ tmin and $t>T f$, the $\mathrm{N}(\mathrm{t}) / \mathrm{N} 0$ is respectively $100 \%$ and $0 \%$-deterministic. Generally, the system of reference is placed at $t$ min, so in this particular case $t \min =0$ and the Eq. (9) can be written as

$$
\operatorname{SMp~N}(\mathrm{t}) / \mathrm{N} 0=\left(\frac{T f-t}{T f}\right)^{p N t}
$$

In this model, $\mathrm{N}(\mathrm{t}) / \mathrm{N} 0$ continuously decreases when $t$ increases, takes values in the closed interval [1;0], and defines the deterministic regions. Its parameters can be obtained from radioactive decay information, but when data are not available; these can be derived from the similarities between Eq. (3) and Eq. (10).

Given the current radioactive decay expression (CRDE) is an EF, which has similarities with the PFs, in particular the SMp N(t)/N0, the SMp can be used in whatever situations employing CRDE, and even replace this model. The use of the SMp model will be more useful, as is shown in the comparison between the CRDE and SMp N(t)/N0 in Table 2, where the differences represent the deficiencies of the CRDE.

The attenuation of radiation is a stochastic process SMp type P3 function of the thickness $x$, so its SMp model is written as

$$
\operatorname{SMp} I(x) / I 0=\left(\frac{x \max -x}{x \max -x \min }\right)^{p I} \quad x \min \leq x \leq x \max
$$

Where $I 0: I(x)$ in $\mathrm{x}=0$, $\mathrm{x}$ min: Maximum value of $x$ for $\mathrm{I}(\mathrm{x}) / \mathrm{I} 0=100 \%$, $x \max$ : Minimum value of $x$ for $\mathrm{I}(\mathrm{x}) / \mathrm{I} 0=0 \%, p I$ : Power of this model $(p I>0), x$ : Thickness 
Table 2. Similarities and differences between the current radioactive decay expression (CRDE) and the SMp $\mathrm{N}(\mathrm{t}) / \mathrm{N} 0)$

\begin{tabular}{|c|c|c|c|}
\hline Aspect & $C R D E$ & SMp N(t)/No & Remarks \\
\hline $\begin{array}{l}\text { Behavior in function of the } \\
\text { independent variable in the } \\
\text { stochastic region }\end{array}$ & Continuously decreases & $\begin{array}{l}\text { Continuously } \\
\text { decreases }\end{array}$ & $\begin{array}{l}\text { Both models have the same behavior, typical } \\
\text { of the SMp type P3 }\end{array}$ \\
\hline $\begin{array}{l}\text { Definition of the stochastic } \\
\text { region; i.e. the interval [tmin; } \\
\text { tmax] }\end{array}$ & Not clearly defined & Well defined & $\begin{array}{l}\text { In the CRDE always } \operatorname{tmin}=0 \text {, while } \operatorname{tmax} \text { or } T f \\
\text { can be obtained by estimation }\end{array}$ \\
\hline Parameters & $T_{1 / 2}$, physical & $\begin{array}{l}\operatorname{tmin} \text { and } \operatorname{tmax}(T f), \\
\text { two physical, and } p K \\
\text { mathematical }\end{array}$ & $T f$ has more familiarization than $T_{1 / 2}$ \\
\hline Physic foundation & It is not good & Very acceptable & $\begin{array}{l}\text { In the CRDE, always } \operatorname{tmin}=0 \text {, and } \mathrm{CRDE}=0 \text { in } \\
\mathrm{t}=+\infty \text {, and these values are same for whatever } \\
\text { radioactive source with finite radioactive } \\
\text { nucleus. }\end{array}$ \\
\hline Probabilistic foundation & $\begin{array}{l}\text { It is not probabilistically } \\
\text { founded }\end{array}$ & Strong & $\begin{array}{l}\text { The CRDE is determined from an unnecessary } \\
\text { solution of a differential equation. }\end{array}$ \\
\hline Math complexity & Middle & Middle & \\
\hline
\end{tabular}

In $x<x \min$ and $x>x \max$, the $\mathrm{I}(\mathrm{x}) / \mathrm{I} 0$ is respectively $100 \%$ - and $0 \%$-deterministic.

Given the current attenuation of radiation expression (CARE) is an EF, which has similarities with the PFs, in particular the SMp I(x)/I0, the SMp model can be used in whatever situations employing CARE, and even replace this model. The use of the SMp model will be superior, since all elements of Table 2 for the current radioactive decay expression are respectively valid for CARE.

Equation (11) determines that a $x \min \neq 0$ is possible for a determined material and radiation, as well as there is a $\mathrm{xmax} \neq \infty$, where for $\mathrm{x}>\mathrm{xmax}, \mathrm{I}(\mathrm{x})=0$.

\subsection{Application of the SMp type P2}

The SMp considers that the Eq. (6) is an unnecessary definition, which is aimed to establish a relationship with the number of radioactive nucleus $\mathrm{N}(\mathrm{t})$ and the decay constant $\lambda$.

In a radioactive source with identical radioactive nucleus $N$, "The number of decays observed over a given interval obeys Poisson statistics" (Patel, 2000), it should be expressed as Eq. (12), and it means there is a mean time with the most likelihood $(\mathrm{tml})$ of occurrence of maximum disintegrations of $N$, just in this time there will be the maximum radioactivity. The number of disintegrations increases for time $\leq t m l$, while this decreases for time $>t m l$.

$$
P D(X=x))=\frac{e^{-\mu} \mu^{x}}{x !}
$$

Where $\mu(t m l)$ : Time with the most likelihood of occurrence of maximum disintegrations, and mean time

$x$ : Disintegration time for the nucleus $N$

The Poisson distribution expression used in (Patel, 2000), is different from Eq. (12) because the number of decays is cumulative in the time. The SMp treats the radioactivity as a SP/E SMp type P2, and considers the definition of Eq. (6) unnecessary. The SMp type P2 was used in (Frometa-Castillo, 2017b) for determining the SMp cell sublethal damage model.

If the system of reference is placed at whatever time $t \geq t m l$ and $t \leq T f$, then the $\mathrm{SMp} \mathrm{A}(\mathrm{t}) / \mathrm{A} 0$ expression is given as

$$
\operatorname{SMp} \frac{\mathrm{A}(\mathrm{t})}{\mathrm{A} 0}=\left(\frac{T f-t}{T f}\right)^{p A} \quad 0 \leq t \leq T f
$$

Where $A 0: \mathrm{A}(\mathrm{t})$ in $\mathrm{t}=0, T f:$ The life time or minimum value of $t$ for $\mathrm{A}(\mathrm{t}) / \mathrm{A} 0=0 \%$ (Tf is the same of the Eq. $(10)), p A$ : Power of this model $(p A>0), t$ : Time 
Given the current radioactivity expression (CRE) is an EF, which has similarities with the PFs, in particular the $\mathrm{SMp} \mathrm{A(t)/A0} \mathrm{of} \mathrm{the} \mathrm{Eq.} \mathrm{(13),} \mathrm{the} \mathrm{SMp} \mathrm{model} \mathrm{can} \mathrm{be} \mathrm{used} \mathrm{in} \mathrm{whatever} \mathrm{situations} \mathrm{employing} \mathrm{the} \mathrm{CRE,} \mathrm{and} \mathrm{even}$ replace this model. The use of the SMp model will be better due to the same reasons of some ones described about the current radioactive decay and attenuation of radiation expressions.

The SMp proposes PFs that will overcome the deficiencies associated with EFs and be able to model the SP/Es with simple and homogeneous probabilistic-mechanistic models using the three types of SP/Es identified by this project. The SMp models will represent new PFs, where the probability of a random variable $\mathrm{X}$ is expressed as $P_{X}=p(y)$, instead of $x$; and the stochastic region is limited by two $0 \%$ and $/$ or $100 \%$-deterministic regions. The SMp treats the radioactivity as a SP/E SMp type P2, and considers unnecessary the definition used for deriving its current expression.

The major deficiency associated to EFs used in the evaluations of SP/Es is that these functions define neither the $100 \%$ - nor the $0 \%$-deterministic region.

Given the EFs used nowadays have similarities with the PFs, in particular the SMp ones; the SMp models can be used in whatever physics example/application employing the EFs, and even replace these functions. Using the SMp will do better physic and probabilistic treatments to the SP/Es.

\section{References}

American Association of Physicists in Medicine. (2012). Report of AAPM TG 166. The Use and QA of Biologically Related Models for Treatment Planning.

Basic Physics of Nuclear Medicine/Attenuation of Gamma-Rays. (2018). In Wikibooks, Open books for an open world. Retrieved April 17, 2018, from https://en.wikibooks.org/wiki/Basic_Physics_of_Nuclear_Medicine/ Attenuation_of_Gamma-Rays

Frometa-Castillo T. (2017b). The Statistical Models Project (SMp) for evaluation of biological radiation effects. American Journal of Applied Mathematics and Statistics, 5(4), 119-124. Retrieved from http://pubs. sciepub.com/ajams/5/4/2/.

Frometa-Castillo, T. (2017a). The Statistical Models Project (SMp) Normal Tissue Complication Probability (NTCP) Model and Parameters. American Journal of Applied Mathematics and Statistics, 5(4) 115-118. Retrieved from http://pubs.sciepub.com/ajams/5/4/1.

Frometa-Castillo, T., \& Frometa-Leon, E. (2017). The statistical models project (SMp) in optimization of radiotherapy treatments. International Journal of Radiology \& Radiation Therapy. Retrieved from http://medcraveonline.com/IJRRT/IJRRT-04-00089.pdf.

International Atomic Energy Agency. (2003). Review of Radiation Oncology Physics: A Handbook for Teachers and Students.

Jones, B., \& Al Morgan, D. (2007). Chapter 4 "Radiotherapy fraction” Radiobiological Modelling in Radiation Oncology. The British Institute of Radiobiology.

Parks, J. E. (2001). Attenuation of radiation. Retrieved from http://www.phys.utk.edu/labs/modphys/Attenuation Radiation.pdf.

Patel, S. B. (2000). Nuclear physics: an introduction (pp. 62-72). New Delhi: New Age International.

\section{Copyrights}

Copyright for this article is retained by the author(s), with first publication rights granted to the journal.

This is an open-access article distributed under the terms and conditions of the Creative Commons Attribution license (http://creativecommons.org/licenses/by/4.0/). 